\title{
MRI scans do not accurately predict hamstring graft diameter for ACL reconstruction
}

\author{
Ricci Plastow \\ Clare Cullen \\ Herbert Imalingat \\ Jamie C Sergeant \\ Neil Jain
}

North Manchester Hospital, Manchester, UK

Corresponding author:

Ricci Plastow

North Manchester Hospital,

Delaunays Road, M8 5RB

Manchester, UK

E-mail: ricciplastow@doctors.org.uk

\section{Summary}

Introduction: Identify whether magnetic resonance imaging (MRI) pre-operatively can help predict intra-operative hamstring graft size for anterior cruciate ligament (ACL) reconstruction. Previous literature has shown correlation but not accuracy or reproducibility of such a prediction.

Methods: 60 pre-operative MR scans from patients who had undergone $A C L$ reconstruction were studied retrospectively. We measured the cross sectional area of gracilis and semitendinosus tendons using MR axial views. The intraoperative graft diameter was recorded. If the diameter intra-operatively was below an $8 \mathrm{~mm}$ threshold the case was highlighted. We then correlated these results with the pre-operative MR measurements. Pearson correlation and BlandAltman plots were used to assess correlation and agreement between the MR and intra-operative measurements.

Results: The Pearson correlation co-efficient was 0.35. The Bland-Altman plots showed poor agreement and reproducibility between readers. We highlighted 6 cases that had a graft diameter below $8 \mathrm{~mm}$. We were able to predict these 6 cases with the pre-operative MR scans producing $100 \%$ sensitivity, but low specificity of $19 \%$.

Conclusion: Pre-operative MR scan measurement of hamstring size is a poor predictor of graft size due to poor specificity and not being reproducible between readers. Therefore, we do not recommend pre-operative MR scan hamstring measurement to predict intra-operative graft size. Level of evidence: IV.

KEY WORDS: ACL, knee, magnetic resonance imaging.

\section{Introduction}

Anterior cruciate ligament $(A C L)$ reconstruction with a four strand hamstring single bundle technique is well documented and used widely throughout the world. However, it is not always possible to obtain a satisfactory graft diameter from the two hamstring tendons. Some studies have suggested grafts smaller than $8 \mathrm{~mm}$ increase the risk of failure. Conte et al. ${ }^{1}$ performed a systematic review of the current literature and found 6.8 times greater relative risk of failure for grafts smaller than $8 \mathrm{~mm}$. As a result the ability to predict graft size pre-operatively could help reduce failure rates, as it may aid in planning graft choice ${ }^{2.3}$. Additionally this would help the surgeon provide informed consent to the patient pre-operatively regarding which graft would be taken during the operation. Several papers have attempted to predict hamstring graft size pre-operatively to facilitate planning for alternative grafts or techniques that may be used. Anthropometric data such as height and weight has been used to try and predict graft size but Camarda et al. ${ }^{4}$ have shown better correlation with radiological data from MR scanning. Table I shows the previous results on prediction of graft size ${ }^{5-9}$. Each has used a Pearson correlation co-efficient to compare MR cross sectional area (CSA) measurements and intra-operative graft diameter.

From these studies the suggestion is that correlation between pre-operative magnetic resonance CSA measurements and intra-operative graft diameter are regarded as being "moderate to good", although one may expect very strong correlation between two measurements of the same quantity.

Pearson correlation analysis can have two sets of measurements that are systematically different but have perfect correlation. Very strong correlation would therefore be required to permit for a predictive 
Table I. Review of current literature on MRI predicting graft size.

\begin{tabular}{llll}
\hline Study & No Pts & $\begin{array}{c}\text { Pearson co-efficient } \\
\text { for CSA of GT and ST }\end{array}$ & Comments on studies \\
\hline Beyzadeoglu et al. 20125 & 51 & $r=0.419$ & 2 levels measured GT + ST 2x magnification \\
Bickel et al. 2008 & 26 & $r=0.641$ & 1 level (Physis) GT + ST no magnification \\
Erquicia et al. 2013 & 33 & $r=0.86$ & 1 level GT + ST 4x magnification \\
Hamada et al. 1998 & 79 & $r=0.697$ & 1 level GT + ST no magnification \\
Wernecke et al. 20119 & 34 & $r=0.53$ & $\begin{array}{l}1 \text { level (Widest point of condyles) } \\
\text { no magnification GT + ST }\end{array}$ \\
\hline
\end{tabular}

CSA, cross sectional area $\mathrm{mm}^{2}$; GT, gracilis tendon; ST, semi tendinosus tendon.

pre-operative tool to be utilised. The previous studies have only used one reader to compare measurements. We therefore have no concept of the reproducibility of the pre-operative MR measurements. These points are crucial in deciding whether to use any technique as a predictive tool in surgical planning.

Our aim was to determine the reproducibility of MR graft measurements between two readers pre-operatively and to produce a measure of accuracy, rather than correlation between the MR measurements and the actual graft size. The previous literature has attempted pre-operative measurements of hamstring diameter and cross sectional area ${ }^{1-6}$. The diameter readings have shown poorer correlation and therefore we have focused on cross sectional area of the tendons as per the existing literature.

We predict that MR scans pre-operatively cannot predict graft size accurately and are not reproducible and should therefore not be used in deciding which graft to use.

\section{Hypothesis}

MR scans pre-operatively cannot predict ACL hamstring graft sizes accurately or be reproduced.

\section{Materials and methods}

We retrospectively reviewed the pre-operative MR scans and operation notes of 60 consecutive patients who underwent $A C L$ reconstruction using an autologous ipsilateral hamstrings graft during a period of two years in one hospital. Cases were excluded if there was an inadequate MR scan, a history of previous hamstring injuries or previous ACL surgery. Two senior surgeons carried out the surgery in one centre. Grafts were harvested using the standard anteromedial incision and tendon stripper. The gracilis and semitendinosus tendons were prepared over a suspensory fixation device to produce a four strand graft that was sutured together using 2 ethi- bond suture with a running locking technique. This graft was measured using $0.5 \mathrm{~mm}$ incremental sizing tubule blocks. The measurement was taken at the largest diameter possible of all 4 tendons when grouped together over the suspensory device. It was measured in this way rather than tendons separately as you want the practical value that needs to pass through the tibial and femoral tunnels. The tendons diameter can change when stressed and fixated. We felt this way would recreate the scenario most likely seen by surgeons. The graft diameter was then converted into CSA for ease of comparison on graphs with the pre-operative measurements. This calculation was: $\operatorname{CSA}=\pi r^{2}$.

\section{MRI technique}

Fat suppressed T2 images in the axial view were obtained for measurement. Two points were used and the average of both readings was calculated. The widest point of the femoral condyles on the axial views and the joint line were measured. These points were the most used landmarks in previous literature and recommended by the musculoskeletal radiologist. One senior surgeon and one radiologist measured the two tendons at both levels and then repeated to produce an average. Figure 1 highlights the technique used.

The PACS (Picture Archiving and Communicating System) region of interest freehand tool was used for CSA. This tool allows the assessor to trace the circumference of the tendon and resultantly the CSA is calculated. The procedure was performed at $x 4$ magnification to improve accuracy of measurements. The mean average CSA of both tendons was added together to give the predictive graft CSA.

This study meets the ethical requirements of this journal.

\section{Statistical methods}

We calculated the Pearson correlation between the pre-operative CSA and intra-operative diameter measurements. We then produced Bland-Altman 


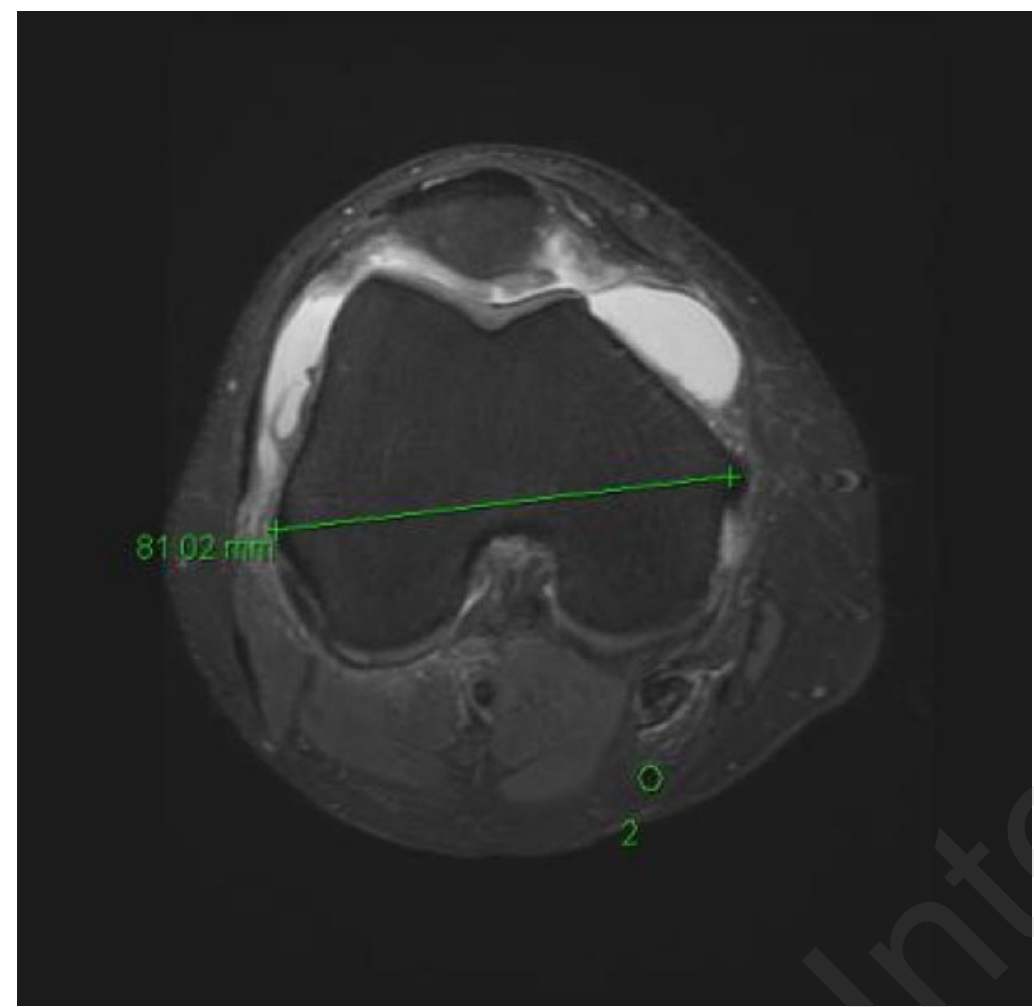

Figure 1. This MR image shows the widest point of the femoral condyles measured and the region of interest tool tracing the semitendinosus circumference.

plots to explore agreement between readers of the pre-operative measurements. The Bland-Altman plots show $95 \%$ limits of agreement, where $95 \%$ of differences in measurements between the readers are expected to exist. This allowed us to decide if the range between data was clinically relevant in predicting graft size. Sensitivity and specificity were also calculated once a cut-off value for pre-operative cross sectional area was decided in order to provide a suitable result for use as a pre-operative surgical planning tool.

\section{Results}

Of the 60 patients, 48 were males and 12 females. The mean age of patients was 29 (range 17-48). Two patients were excluded due to being revision cases. The mean diameter for the hamstring graft intra-operatively was $8 \mathrm{~mm}$ once sutured and suspended. The pre-operative mean CSA was $42 \mathrm{~mm}^{2}$ and intra-operative mean CSA was $50 \mathrm{~mm}^{2}$.

\section{Pearson correlation}

The Pearson correlation co-efficient score of 0.35 was between the pre-operative CSA for both tendons and the intra-operative diameter of the graft (Fig. 2). You can see there is very little linear correlation of values.

\section{Bland-Altman plot analysis}

We have used Bland-Altman plots to visualise the difference between the measurements of the two read- ers and between the pre-operative MR scans and intra operative graft sizes. We have added the upper and lower limits of agreement. These show two standard deviations above and below the mean. If there is a large difference between these it suggests there is poor agreement between the two readers and the MR predictions compared to the intra-operative graft size. Figure 3 shows significant variation in measured tendon size between the two readers. The limits of agreement are far apart with over $10 \mathrm{~mm}^{2}$ either side of the mean.

\section{Agreement between pre-operative MR and intra- operative graft size}

Our aim was to determine whether the MR scan measurements pre-operatively are suitable in predicting intra-operative graft size. Pearson correlation co-efficient scores cannot achieve this. Bland-Altman plots visually permit us to see the agreement between data and provide $95 \%$ limits of agreement. If the upper and lower limits of agreement are significantly apart then we may conclude the differences are too large to predict graft size in this instance.

Figure 4 shows that MR measurements on average were $7 \mathrm{~mm}^{2}$ lower than intra-operative CSA graft sizes with limits of agreement ranging between 15 $\mathrm{mm}$ and $-30 \mathrm{~mm}^{2}$.

Using a cut off value of $46 \mathrm{~mm}^{2}$ pre-operatively for graft cross sectional area we would be able to produce $100 \%$ sensitivity $(95 \% \mathrm{Cl} 54-100)$ for providing a graft of at least $8 \mathrm{~mm}$ in diameter. However the specificity for this was $19 \%(95 \% \mathrm{Cl} 12-51)$. 


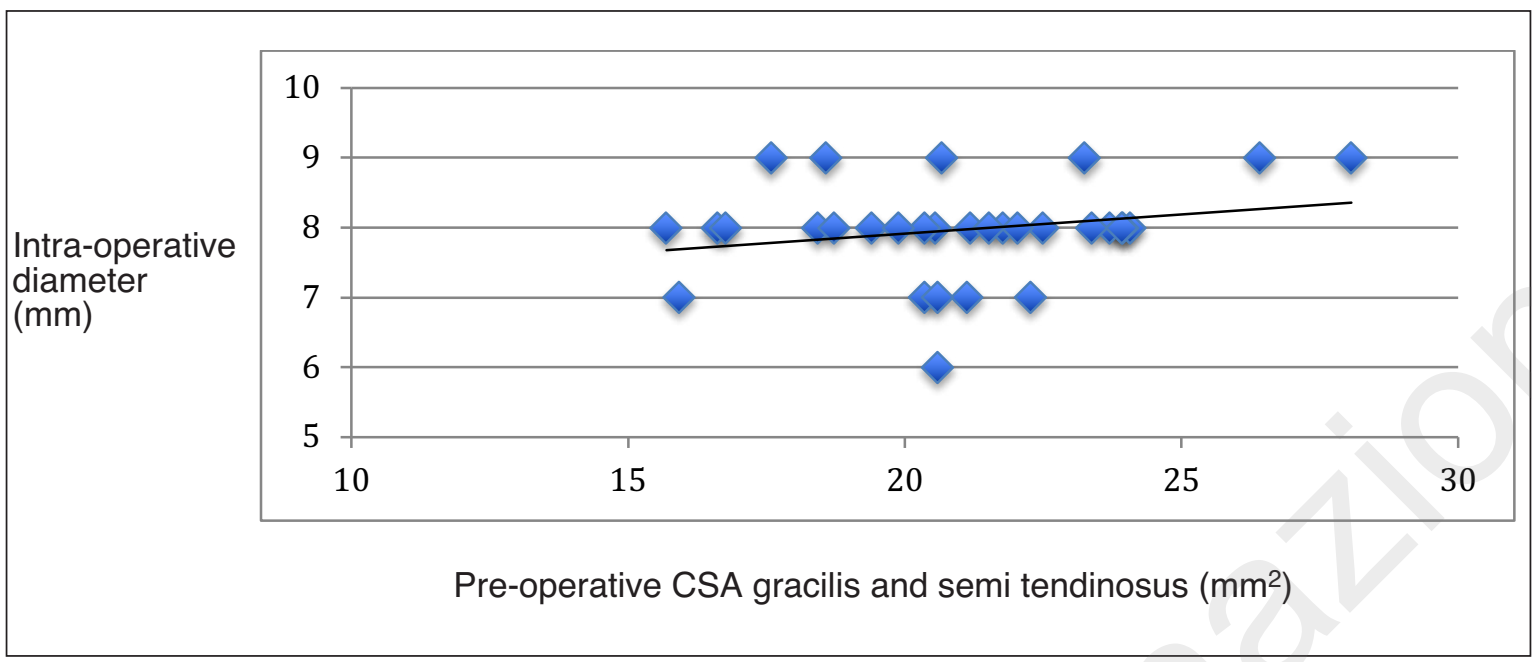

Figure 2. Pearson correlation graph comparing pre-operative CSA and intra-operative graft size.

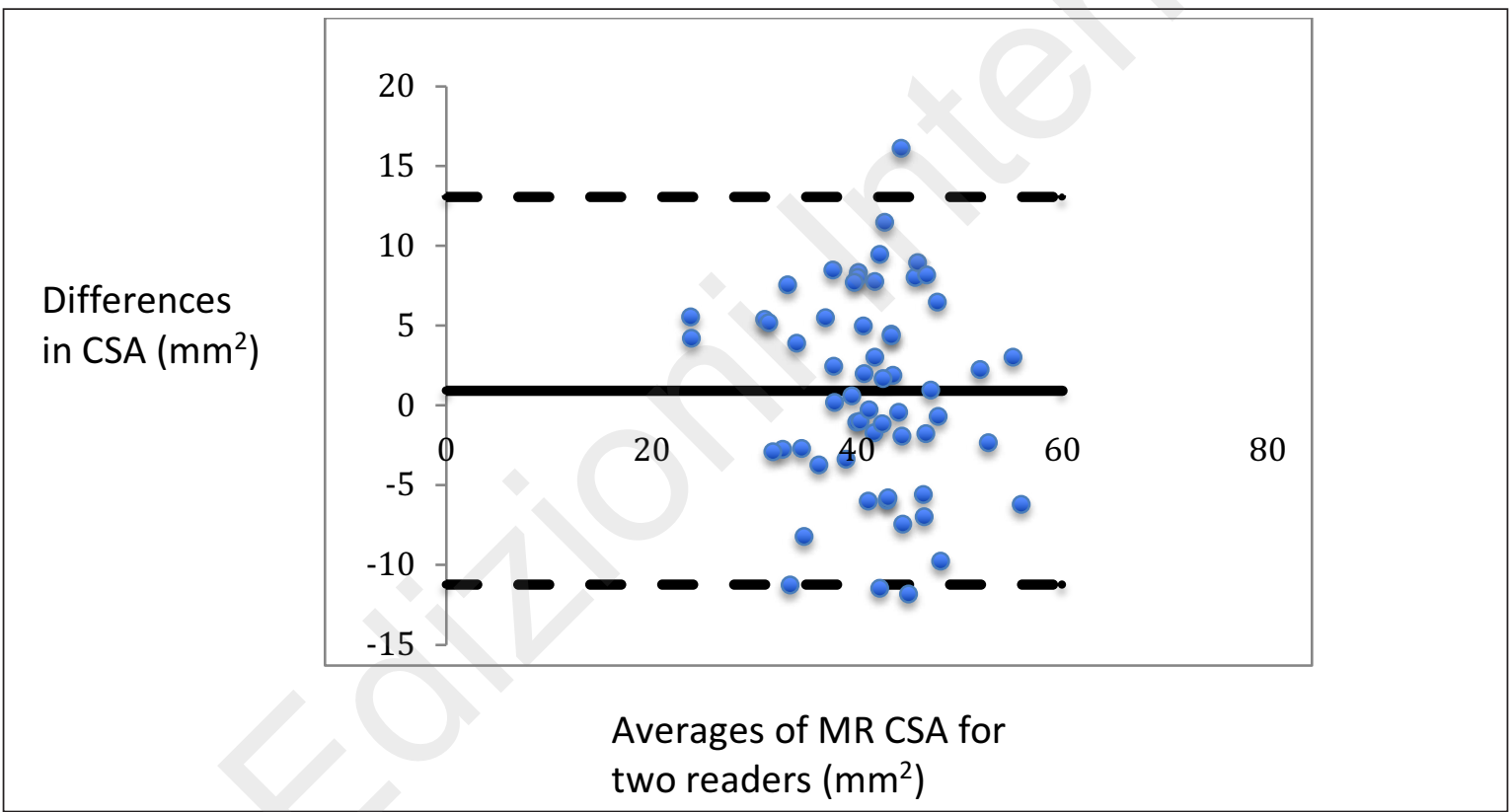

Figure 3. Bland-Altman plot comparing two readers pre-operative measurements.

\section{Discussion}

Our Pearson correlation score of 0.35 is lower than the previous literature as shown in Table I. Following critical review of these papers the highest correlation score of $0.86^{4}$ was achieved with only one surgeon and only one measurement at two times magnification. The 0.4 score seen in one paper $^{2}$ used a more robust technique of four times magnification and repeat measurements similar to our technique. We therefore feel our correlation score is more likely to represent a true reflection of this process of MR prediction of graft size.

We believe the clinically relevant issue is accuracy and reproducibility of the MR scan prediction over correlation. One would expect very strong correlation between two measurements of the same quantity. To have such varying correlation across the literature would suggest a reproducibility and accuracy problem or a variation in the techniques used in order to determine graft size.

Our further analysis using Bland-Altman plots investigated the reproducibility between readers and wide variation between pre-operative CSA and intra-operative diameter. The $95 \%$ limits of agreement show the outlier measurements are not acceptable when deciding whether the graft pre-operatively is sufficient or not. This could potentially be corrected with use of an equation to create a predictive tool but unfortunately the limits of agreement would remain too broad as they range from nearly $15 \mathrm{~mm}^{2}$ to $-30 \mathrm{~mm}^{2}$. These values make a significant clinical difference to predicting a graft diameter. 


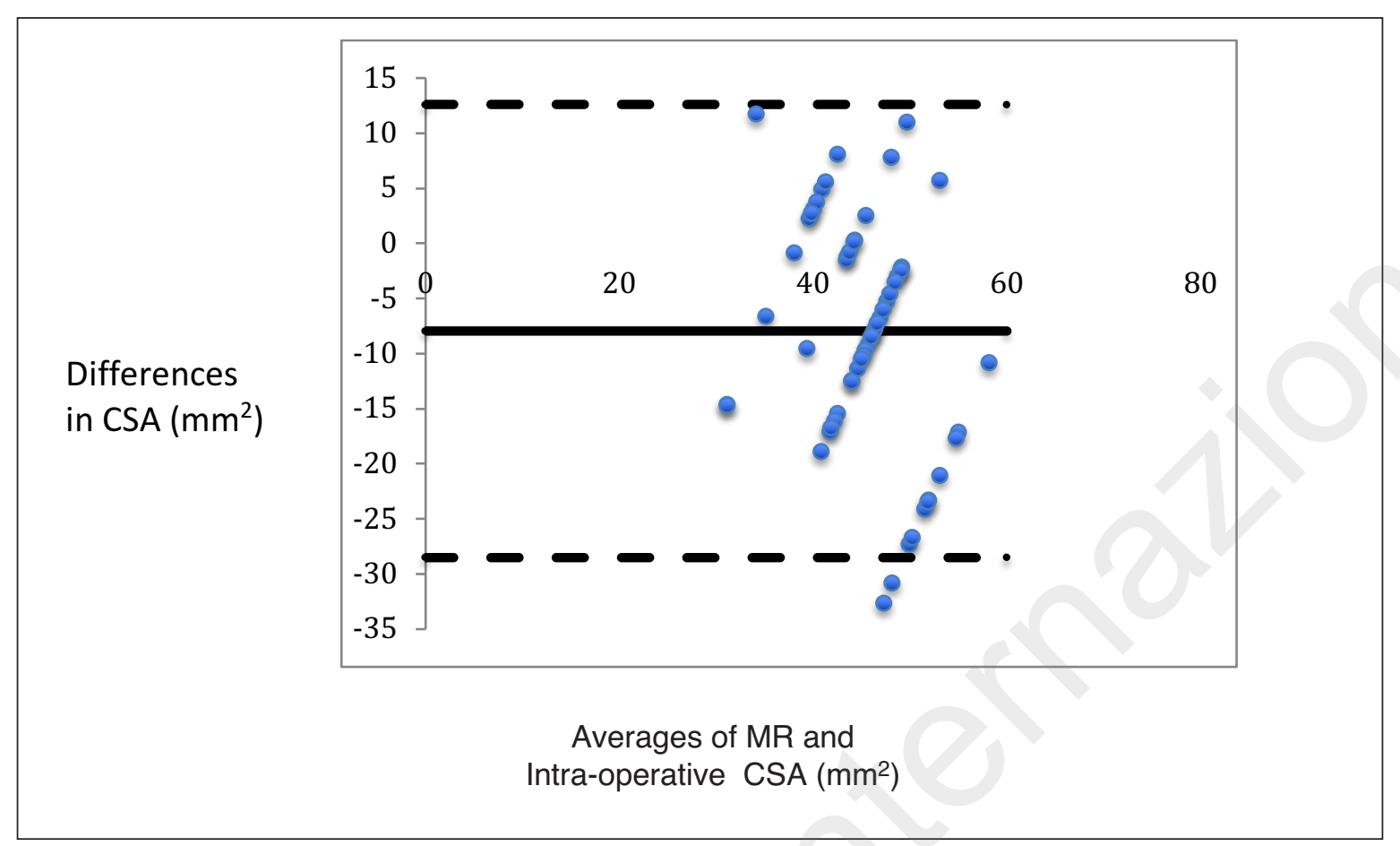

Figure 4. Bland-Altman plot comparing pre-operative CSA measurements and intra-operative graft sizes.

Even with these large differences we decided to create a cut off value of $46 \mathrm{~mm}^{2}$ for the MR scan measurements of cross sectional area. This allowed us to create sensitivity and specificity values for the MR predictive tool. The specificity of $19 \%$ is very poor and highlights that this predictive tool cannot be used. The purpose of creating a cut off threshold would be to aid surgical planning. Using this value would see surgeons unnecessarily change their plans on a regular basis. This cut off value could be increased but that would create a large number of false negative results. This would mean surgeons still found grafts smaller than $8 \mathrm{~mm}$ despite using the predictive tool.

We are the only study to have more than one observer measuring pre-operative MR graft size. We used one surgeon and one radiologist to measure the tendons on MR scans and one surgeon to measure intra-operatively. It is impossible to assess how reproducible pre-operative MR measurements are from the previous literature with only one observer.

The limitations of this study include the low number of patients, use of computer tools to measure tendons, MR accuracy and $0.5 \mathrm{~mm}$ incremental blocks for measuring graft sizes. The landmarks for measurement on MR also do not correlate exactly with where we measured the graft intra-operatively. The two variables of surgeon and MR accuracy are impossible to calculate into a simple predictive tool. Adding pre-operative CSA measurements together to compare to intra-operative graft diameter is not sufficient. The practical nature of tendon harvest and graft preparation where the tendons are stripped, sutured and wrapped together will change their dimensions and therefore ultimately decrease such accuracy.

\section{Conclusions}

We cannot recommend use of pre-operative MR scan measurement of gracilis and semitendinosus diameter or cross-sectional area to predict hamstring graft size for $A C L$ reconstruction.

\section{Compliance with ethical standards}

\section{Conflict of interest}

There were no conflicts of interest for any Author in this study.

\section{Acknowledgements}

Jamie Sergeant.

\section{Ethics}

The Authors declare that this research was conducted following basic ethical aspects and international standards as required by the journal and recently update $\mathrm{in}^{10}$.

\section{References}

1. Conte E, Hyatt A, Gatt C, Dhawan A. Hamstring Autograft Size Can Be Predicted and Is a Potential Risk Factor for Anterior Cruciate Ligament Reconstruction Failure. Arthroscopy. 2014;30(7):882-885. 
2. Charalambous CP, Kwaees TA. Anatomical considerations in hamstring tendon harvesting for anterior cruciate ligament reconstruction. MLTJ. 2012;2(4):253-257.

3. Drocco L, Camazzola D, Ferracini R, Lustig S, Ravera L, Graziano E, et al. Tripled semitendinous with single harvesting is as effective but less invasive compared to standard gracilissemitendinous harvesting. MLTJ. 2017;7(4): 564-572.

4. Camarda L, Grassedonio E, Albano D, Galia M, Midiri M, D'Arienzo M. MRI evaluation to predict tendon size for knee ligament reconstruction. MLTJ. 2017;7(3):478-484.

5. Beyzadeoglu T, Akgun U, Tasdelen N, Karahan M. Prediction of semitendinosus and gracilis autograft sizes for ACL reconstruction. Knee Surg Sports Traumatol Arthroscopy. 2012; 20:1293-1297.

6. Bickel BA, Fowler TT, Mowbray JG, Adler B, Klingele K, Phillips G. Preoperative magnetic resonance imaging crosssectional area for measurement of hamstring autograft diameter for reconstruction of the adolescent anterior cruciate ligament. Arthroscopy. 2008;24:1336-1341.
7. Erquicia JI, Gelber PE, Doreste JL, Pelfort X, Abat F, Monllau JC. How to improve the prediction of quadrupled semitendinosus and gracilis autograft sizes with magnetic resonance imaging and ultrasonography. Am J sports Med. 2013;41: 1857-1863.

8. Hamada M, Shino K, Mitsuoka T, Abe N, Horibe S. Cross-sectional area measurement of the semitendionsus tendon for anterior cruciate ligament reconstruction. Arthroscopy. 1998 14:696-701.

9. Wernecke G, Harris IA, Houang MT, Seeto BG, Chen DB, Macdessi SJ. Using magnetic resonance imaging to predict adequate graft diameters for autologous hamstring doublebundle anterior cruciate ligament reconstruction. Arthroscopy. 2011;27:1055-1105.

10. Padulo J, Oliva F, Frizziero A, Maffulli N. Muscles, Ligaments and Tendons Journal - Basic principles and recommendations in clinical and field science research: 2016 update. MLTJ. 2016;6(1):1-5. 